30. september 20:

19:10 


\title{
High prevalence of ADHD symptoms in unmedicated youths with po narcolepsy type 1
}

\author{
Berit Hjelde Hansen ${ }^{1}$, Hilde T Juvodden ${ }^{1,2}$, Sebjørg Hesla Nordstrand ${ }^{2,}$ \\ Rannveig Viste ${ }^{1,2}$, Per M Thorsby ${ }^{4}$; David Swanson ${ }^{5}$, Kristian B Nilsen ${ }^{6}$, \\ Nærland ${ }^{1,2}$, Stine Knudsen-Heier ${ }^{1}$
}

1 Norwegian Centre of Expertise for Neurodevelopmental Disorders and Hypersomnias, of Rare Disorders, Division of Paediatric and Adolescent Medicine, Oslo University Hospi 2 Institute of Clinical Medicine, University of Oslo, Norway

3 Department of Pediatrics, Division of Pediatric and Adolescent Medicine, Oslo Universi Ullevål Hospital, Norway

4 Hormone Laboratory, Department of Medical Biochemistry, Oslo University Hospital, A Oslo, Norway

5 Oslo Centre for Biostatistics and Epidemiology, Oslo University Hospital

6 Department of Neurology, Section for Clinical Neurophysiology, Oslo University Hospit Hospital, Norway

Corresponding author: Berit Hjelde Hansen, Norwegian Centre of Expertise for Neurode Disorders and Hypersomnias, P.B. 4956, Nydalen, 0424 Oslo, Norway

E-mail: 


\begin{abstract}
Objectives To characterize attention deficit-hyperactivity disorder (ADHD) sympt unmedicated post-H1N1 narcolepsy type 1 (NT1) youths, and explore association ADHD symptoms and the narcolepsy phenotype.

Methods Fifty consecutively enrolled post-H1N1 NT1 youths ( $7-20$ years, $62 \%$ fer HLA-DQB1*06:02-positive, $98 \%$ CSF hypocretin-1 deficient, $88 \%$ vaccinated) wer after two weeks off medication for ADHD (ADHD diagnosis pre/post-narcolepsy rated ADHD symptoms) and narcolepsy-phenotyped (semi-structured interview, Sleep Questionnaire, Epworth Sleepiness Scale, polysomnography (PSG), Multiple Latency Test (MSLT)).

Results $26(52 \%)$ and 15 (30\%) of participants had ADHD symptoms above and be clinical significant cut-off, respectively, while $9(18 \%)$ had no ADHD symptoms. Hi were found for ADHD total score (mean (SD), 17.9 (9.5)) and ADHD subscores (ini score, 11.0 (6.3); hyperactive/impulsivity score, $6.9(4.7))$. These were significant than previously reported in a mainly medicated narcolepsy cohort $(p<0.0001)$. A and disease duration did not influence scores. Two participants (4\%) had ADHD , prior to narcolepsy onset. ADHD symptoms were correlated with parent-rated, b patient rated ESS scores, objective sleepiness (mean sleep latency), sleep fragme (sleep stage shift index, awakening index), or CSF hypocretin-1 level.
\end{abstract}

Conclusion Comorbid ADHD symptoms were more prevalent in unmedicated pos 
narcolepsy sleep/wake phenotype characteristics, indicating that the ADHD symF not a direct consequence of disturbed sleep or daytime sleepiness.

Keywords: ADHD symptoms, narcolepsy, H1N1

\section{Abbreviations:}

ADHD Attention Deficit- Hyperactivity Disorder

NT1 narcolepsy type 1

NT2 narcolepsy type 2

PSG polysomnography

MSLT Multiple Sleep Latency Test

CSF cerebrospinal fluid

Hcrt-1 hypocretin-1

ICSD-3 International Classification of Sleep Disorders $3^{\text {rd }}$ version

SDB sleep disordered breathing

PLM periodic limb movement

AASM American Academy of Sleep Medicine

ADHD RS ADHD rating scale

TST total sleep time

SSQ Stanford Sleep Questionnaire

ESS Epworth Sleepiness Scale 
$\mathrm{CV}$ coefficient of variation

SD standard deviation

ASEBA Achenbach System of Empirically Based Assessment

CBCL Child Behavior Checklist

ASR Adult Self Report

$\mathrm{HH}$ hypnagogic hallucinations

SP sleep paralysis

AHI apnea/hypopnea index

SL sleep latency

MWT maintenance of wakefullness test

RLS restless legs syndrome

REM rapid eye movement

SSRI selective serotonine reuptake inhibitor 


\section{INTRODUCTION}

Narcolepsy is a chronic neurological disorder affecting the sleep-wake and muscl regulatory systems in the brain (1). Core narcolepsy symptoms consist of excessi sleepiness, disrupted night sleep and sleep fragmentation, cataplexy (sudden los: tone typically triggered by strong emotions), sleep paralysis, and hypnagogic and hypnopompic hallucinations According to the $3^{\text {rd }}$ edition of the International Cla: of Sleep Disorders (ICSD-3) (2), narcolepsy type 1 (NT1) is differentiated from nal type 2 (NT2) by the presence of cataplexy and/or a low level of the neuropeptide 1 in the cerebrospinal fluid (CSF Hcrt-1 levels). The hypocretin-producing cells in 1 hypothalamus project widely in the brain and strongly to the brain stem and thal producing an excitatory effect on wake- and arousal-promoting nuclei, including involved in noradrenergic and dopaminergic transmission (3). Destruction of hyp producing neurons in the hypothalamus is considered a key pathophysiological $\mathrm{p}$ NT1 (1). The prevalence of sporadic narcolepsy is estimated to be $25-50$ cases pe individuals $(4,5)$. Following the $2009 \mathrm{H} 1 \mathrm{~N} 1$ epidemic and vaccination campaign $\mathrm{v}$ Pandemrix ${ }^{\circledast}$ vaccine, there was an abrupt increase in the incidence of NT1 in Nor other countries (6-8).

The neurodevelopmental disorder Attention Deficit Hyperactivity Disorder (ADHI characterized by symptoms of inattention, impulsivity and hyperactivity. These s! and especially so of inattention are frequently reported in adults and children wit narcolepsy (9-12), and it is associated with reduced quality of life, academic failuı 
pathophysiological mechanism (15). Sleep disturbances are a frequent comorbidi (16-18) and may precipitate and aggravate the ADHD symptoms $(19,20)$. Multipl dysfunctions may underpin the behavioral symptoms of ADHD, including difficult sustained attention, response inhibition, working memory, and executive functio necessary for attention regulation, planning and execution of tasks, self-regulatic monitoring)(19, 21). Gender and age trends indicate that symptoms are more fre boys than in girls, and that ADHD symptom scores are higher in younger than in c children (22).

The exact "true" prevalence of ADHD symptoms in pediatric narcolepsy is unkno the majority of young people considered in the previous studies were taking som medication for their narcolepsy at the time their ADHD symptoms were measure 12). Many of these medications are the treatments of choice for ADHD (23). One reported ADHD symptoms above the clinical cut-off in almost $20 \%$ of a sample of with NT1 (87\% sporadic, $67.1 \%$ were taking medication, ADHD assessed in $61 \mathrm{ch}$ another study of 38 children with narcolepsy ( $18.4 \%$ sporadic, $94.7 \%$ were takin medication, ADHD scores assessed in 32 children) more than one in four met crit ADHD diagnosis (10). Finally, an Italian study reported higher ADHD scores in chil narcolepsy (72\% medicated) compared with controls (12).

The mechanisms driving the high levels of occurrence of ADHD symptoms in narc unknown. One explanation may be that pre-existing ADHD represents vulnerabili development of narcolepsy in the face of particular environmental triggers. For it 
narcolepsy are due to a low level of Hcrt-1 $(9,25)$, or to be a consequence of slee sleep fragmentation $(9,20,25,26)$. However, empirical support for these explani scarce. Associations between ADHD symptoms and CSF Hcrt-1 levels in narcoleps been extensively studied. One pediatric, and largely sporadic study reported no s association, although very few had their CSF Hcrt-1 levels measured (9), and two sporadic adult populations did not report any significant difference in ADHD sym between with NT1 (low CSF Hcrt-1 levels) and NT2 (normal CSF Hcrt-1 levels)(26 Results are not consistent regarding the associations between ADHD symptoms $\hat{c}$ sleepiness. One pediatric study reported an association between ADHD symptor subjective daytime sleepiness, while no associations were found between ADHD and objective measures of sleepiness. However, the conclusions were limited ber mostly medicated and there was an average of 1.2 years between ADHD sympto। assessment and the sleep investigations(9). No significant correlations between $t$ symptoms and subjective or objective daytime sleepiness were found in unmedic with a primary central hypersomnolence disorder (26), while a positive correlatic ADHD scores and subjective daytime sleepiness was reported from 21 unmedicat NT1 (27). To our knowledge, associations between ADHD symptoms and measur fragmentation have not previously been examined in the context of narcolepsy.

The aim of this study is to characterize ADHD symptoms in a sample of unmedica H1N1 NT1 young people, defined as youths with narcolepsy type 1 with debut af 
objective narcolepsy phenotypic features (CSF Hcrt-1 levels, subjectively reporter sleepiness, multiple sleep latency test (MSLT) mean sleep latency, polysomnogra sleep fragmentation). As sleep-disordered breathing (SDB) and periodic limb mol (PLM) symptoms have both been associated with ADHD symptomatology (16, 17 wanted to adjust for possible associations between ADHD symptoms and PSG m€ SDB and PLM symptoms. Lastly, we wanted to explore whether ADHD symptoma narcolepsy is due to ADHD existing prior to narcolepsy onset, or family aggregati، or other neurodevelopmental disorders, likely to be associated with high levels o symptoms even in the absence of a formal ADHD diagnosis (28).

We hypothesized that: our sample of unmedicated post-H1N1 NT1 youths would ADHD symptom scores; inattentive symptoms in our group would be significantl) frequent than hyperactive/impulsive symptoms; and ADHD scores would be signi higher than previously reported in mainly medicated youths with narcolepsy. We priori hypothesis about any association of ADHD symptoms with a pre-existing AI diagnosis or family aggregation of neurodevelopmental disorders, CSF Hcrt-1 lev measures of daytime sleepiness, or sleep fragmentation.

\section{METHODS}

\subsection{Participants}

Following the increase in narcolepsy incidence in Norway after the 2009 H1N1 epi 
and Care Services to register and follow up all patients with narcolepsy onset after 2009 in Norway, irrespective of their vaccination status.

Between February 2015 and June 2017, after obtaining written consent, 63 NT1 yc years old) were consecutively enrolled at our center. We have previously reported population partially overlapping our study population. The large majority of partici had previously been treated, hence were not medication naïve. All participants we instructed to be unmedicated with their narcolepsy medication and all other medi affecting sleep for two weeks prior to inclusion in accordance with recommendatic American Academy of Sleep Medicine (AASM) (29). Furthermore, participants on anticataplectic medication were instructed to taper these off over 1-2 weeks (dept dosage) prior to the two unmedicated weeks. All participants complied with these instructions, except one who was allowed to remain on venlafaxine due to severe The majority - 34/50 (68\%) - of participants attended school in the unmedicated p with some adjustments. A total of 14/50 (28\%) were included during a school vac period and 2/50 (4\%) did not attend school in the two week period prior to asses Comparison between the group attending school versus those included in vacatic not attending school of ADHD rating scale (RS) total, inattentive, and hyperactive scores revealed no significant differences $(p=0.519 ; p=0.747 ; p=0.392$, respecti of the 63 youths $(79.4 \%$ ) had an ADHD (RS) provided by their parents. There wer significant differences in demographic or clinical sleep variables between the 13 ; 
group with parental ADHD RS (mean (SD) TST, 6.5 (2.1) vs. 8.0 (1.0); $p=0.005$ ). TI with no ADHD RS available were excluded from further analyses.

The study was approved by the Norwegian regional committees for medical and I research ethics (REK).

\subsection{Procedure}

Participants were instructed to wear an actigraph (Actiwatch Spectrum Plus, Phill days before inclusion (e.g. during the unmedicated period). Following admission center, semi-structured interviews, and questionnaires (Stanford Sleep Question including the Epworth Sleepiness Scale (ESS) (patient- and parent-rated)) (30), an (parent-rated) were administered (31). Sleep investigations (PSG, MSLT) were col and blood samples taken to determine routine parameters and carry out HLA ani was done in order to verify and validate the narcolepsy diagnose according to the and to provide a detailed description of the sleep phenotype as part of our speci; assignment, and also to - for research and comparable purposes - ensure that th investigations were performed with the same method for all participants. All und overnight PSG followed by an MSLT with five 30-minute nap opportunities. Techr of the PSG/MSLT procedure have been published previously (32). All sleep recor scored by two skilled sleep technicians/nurses, who scored the sleep stage and a events according to AASM criteria (33). The clinical and sleep parameters were e' an experienced neurologist and sleep medicine expert (SKH) using the ICSD-3 crit diagnoses (2), including the verification of the NT1 diagnoses. 
previously described (34). Limit of detection (LOD) was $20 \mathrm{pg} / \mathrm{ml}$ and limit of qu (LOQ) $40 \mathrm{pg} / \mathrm{ml}$ where the coefficient of variation (CV) was $20 \%$. At $109 \mathrm{pg} / \mathrm{ml} \mathrm{t}$ the method was $9 \%$. Lumbar punctures for sampling CSF were performed at loc before (in $88 \%$ of cases) or after study inclusion, in average 3.3 years (range 0 . years) prior to inclusion or in average 0.3 years (range 0.08 to 0.4 years) after assessment.

\subsection{Measures}

\subsubsection{Present $A D H D$ symptoms}

were assessed by parental ratings of ADHD symptoms on the ADHD RS as occurri unmedicated period. The ADHD RS is a questionnaire measuring severity of the $A$ symptoms inattention, hyperactivity and impulsivity, and has been widely used ir clinical populations, including in narcolepsy populations (9). It covers the 18 sym ADHD according to the Diagnostic and Statistical Manual of Mental Disorders $5^{\text {th }}$ rated on a scale comprising values of 0 (never), 1 (sometimes), 2 (often), and 3 ( $v$ (31) and for which good psychometric properties have been reported, including $f$ Norwegian version (35-37). Two subscale scores were calculated: the inattentive hyperactivity/impulsivity scores, in addition to the ADHD total score, which is the 18 items. A higher score indicates more ADHD symptoms. There are no Norwegic population norms, and the presence of ADHD symptoms above the clinical cut-of determined according to Danish population norms based on age- and gender-stri 
populations. A symptom of ADHD was considered to be present if the item was $g$ of 2 or more. The youths were divided into three groups based on their ADHD sCI clinical scores (above the clinical cut-off according to the Danish group norms), A symptoms (at least one item score of 2 or more, sum score below the clinical cut ADHD symptoms (no item score above 1). Internal reliability of our sample was g values of Cronbach's alpha of: $0.87,0.89$ and 0.75 for the total, inattentive and hyperactive/impulsivity scales, respectively. These results are comparable to tho: in clinical ADHD populations (40).

\subsubsection{ADHD diagnosis pre narcolepsy}

ADHD diagnose pre narcolepsy debut was assessed by patient chart review.

\subsubsection{Family history of neurodevelopmental disorders}

Family history of neurodevelopmental disorders was assessed by parental report interview with a questionnaire assessing familial occurrence of somatic and psycl disorders.

\subsubsection{Other psychiatric symptoms}

Our center has previously reported psychiatric symptoms in a sample of children with narcolepsy type 1 (41). In this study the Norwegian version of the Achenbac Empirically Based Assessment (ASEBA) was used; the parent-rated Child Behavio $(\mathrm{CBCL})$ for age 6-18 years, and the corresponding Adult Self Report (ASR) for ag 
(anxious/depressed, withdrawn/depressed and somatic complaints) and an exte। score (rule-breaking behavior, aggressive behavior and intrusive behavior (adult . only)). The other syndrome scales (thought problems, attention problems and so problems (child version only)), are used in conjunction with the internalizing or $\epsilon$ scores to derive a total score. Details on assessment and scoring have previously published (41).

\subsubsection{Subjective sleepiness}

taken as that occurring during the unmedicated period (days 15-16), was assesse report and parental report on the ESS, which yields scores ranging from 0 to 24 . I of 11 or more are considered to indicate increased daytime sleepiness (45). ESS s was available for all 50 participants with parental ADHD RS report, and parent-re was available for $43(86 \%)$ of these participants.

\subsubsection{Other core narcolepsy symptom}

Cataplexy, hypnagogic hallucinations $(\mathrm{HH})$, and sleep paralysis (SP) prevalences a frequencies were assessed at the end of the unmedicated period with the SSQ ( 3

\subsubsection{Objective sleepiness and sleep fragmentation.}

Mean MSLT SL was used as a measure of objective daytime sleepiness. Sleep frą was assessed by the sleep stage shift index and awakening index (measured as th

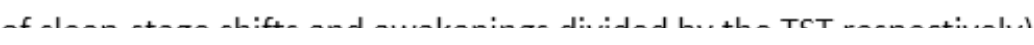




\subsubsection{SDB and PLM}

were measured by the apnea/hypopnea index $(\mathrm{AHI})$ and periodic limb movemen index (measured as the number of apnea/hypopnea events and periodic limb mc divided by the TST respectively).

\subsection{Statistics}

Data were analyzed with IBM SPSS version 23. Variables are summarized as frequ the mean and SD, as appropriate. We tested the independence of pairs of catego variables using chi-squared $\left(\mathrm{X}^{2}\right)$ hypothesis tests, and for continuous variables, $\mathrm{w}$ parametric tests (Mann-Whitney for independent samples, Wilcoxon's signed-ra। paired samples, Kruskal-Wallis for three or more samples, and Spearman's rho to correlation) (46), to prevent undue influence of outliers. We wanted to compare with previously reported ADHD scores from a sample of predominantly medicate with NT1 (9), an ADHD sample (40), and a healthy control group (40). We first tra the confidence intervals of the ADHD RS scores from the pediatric NT1 sample (A known for $61 / 86)$ to SDs using the formula: $\mathrm{SD}=(\mathrm{C} 2-\mathrm{m}) / 1.96$, where $\mathrm{C} 2$ is the $\mathrm{u}$ | quantile from a $95 \%$ confidence interval, and $\mathrm{m}$ is the group mean. We then com means with a two-sample t-test (https://www.graphpad.com/quickcalcs/ttest1). Multiple linear regression analyses, adjusted for age, gender, PLM index and AHI, carried out to assess the association between ADHD total score, and scores for $\mathrm{p}_{\mathrm{i}}$ self-reported ESS, mean MSLT sleep latency (SL), sleep stage shift index, and awa 
0.05 were considered statistically significant, unadjusted for multiple comparisor exploratory nature of study.

\section{RESULTS}

\subsection{Characteristics}

Demographic and clinical data of the 50 participants, including sleep parameters summarized in Table 1. A majority (62\%) of participants were female. CSF Hcrt-1 available for 49 (98\%) of the participants with exact values known for $44(88 \%)$ o Levels were further categorized into groups: $<40 \mathrm{pg} / \mathrm{ml}(\mathrm{n}=23), 40-150 \mathrm{pg} / \mathrm{ml}(\mathrm{n}=$ "low" (defined as "low" by the laboratory, but with no exact value available, $n=5$ )

Please insert table 1 about here.

The self-reported and parent-reported ESS were significantly positively correlate $0.66, p<0.001)$. Ninety-eight percent of participants $(49 / 50)$ were HLA DQB1*06 Mean MSLT SL was 3.3 minutes. Forty-six out of 50 participants were cataplectic. participants $(8 \%)$ - all hypocretin deficient - were cataplexy negative. The majori participants exhibited $\mathrm{HH}(82 \%)$ and SP (64\%). There was an average of 14.4 sleel shifts / hour TST, and 2.5 awakenings / hour TST. The mean AHI was below the di threshold for sleep apnea both for adult $(<5 /$ hour TST) and pediatric criteria $(<1$ while the PLM index was high (17.0/hour TST). 


\subsection{ADHD symptoms in NT1}

$26 / 50(52 \%)$ of the young people had levels of ADHD symptoms above the cut-of to the Scandinavian (Danish) age- and gender-adjusted norms, 15 (30\%) had one ADHD symptoms but with summed scores below the clinical range, and $9(18 \%) \mathrm{r}$ ADHD symptoms, i.e. no item score greater than 1.

There was no significant difference in the proportions of the sexes among the thr of ADHD symptom loads (ADHD clinical scores: $61.5 \%$ female; ADHD symptoms: : female; no ADHD symptoms: $44.4 \%$ female; $\left.\mathrm{X}^{2}=2.0, p=0.362\right)$. Youths with these levels of ADHD symptom loads did not differ with respect to age at inclusion (AD) scores 14.7 years (3.0); ADHD symptoms: 16.2 years (3.0); no ADHD symptoms: 1 (4.0); $p=0.255)$, age at disease onset (ADHD clinical scores: 9.3 years (3.1); ADH[ symptoms: 11.3 (3.0); no ADHD symptoms: 9.5 years (3.3); $p=0.205)$, disease $d u$ (ADHD clinical scores: 5.4 years (1.3); ADHD symptoms: 4.9 years (1.7); no ADHD 4.6 years (1.5); $p=0.217$ ), -or age at H1N1 vaccination (ADHD clinical scores: 8.5 , ADHD symptoms: 9.8 years (3.3); no ADHD symptoms: 8.7 years $(3.9) ; p=0.592$ ). there were few participants without ADHD symptoms $(n=9)$. Mean (SD) ADHD RS scores were: total score, 17.9 (9.5); inattentive score, 11.0 ( $f$ hyperactivity/impulsivity score: 6.9 (4.7). There were no differences in the ADHD subscale scores by gender; ADHD total score: girls 12.7 (7.8) versus boys 19.1 (11 0.484; inattentive score: girls 10.5 (5.0) versus boys 11.7 (8.1), $p=0.603$; hyperactive/impulsivity score: girls 6.6 (4.2) versus boys 7.4 (5.5), $p=0.764$. 
hyperactivity/impulsivity scores were not significantly correlated with age at inclı at disease onset, age at vaccination, or disease duration (data not shown). ADHD inattentive scores were significantly higher than hyperactivity/impulsivity : (11.0 (6.3) versus $6.9(4.7)$ respectively, $p<0.001)$.

As shown in Figure 1, the ADHD RS total score in our unmedicated NT1 sample w significantly higher than that previously reported from mainly medicated childreI (17.9 (9.5) vs. $12.2(1.6) ; t=4.612, p<0.0001)$ (9). Our subscores were also highel those reported in a healthy control group (inattentive subscore, 11.0 (6.3) vs. 4.1 9.274, $p<0.0001$; hyperactive/impulsivity subscore, 6.9 (4.7) vs. $3.4(3.4) ; t=5.5$ 0.0001 ) (40), but lower than those reported in an ADHD sample (inattentive subs (6.3) vs. 17.9 (5.5), $t=8.359, p<0.0001$; hyperactive/impulsivity subscore, 6.9 (4 (7.1), $t=5.075, p<0.0001)(40)$.

Please insert figure 1 about here (one column)

\subsection{ADHD diagnose prior to narcolepsy debut and family history of neurodevelop} disorders In two participants (4.0\%) an ADHD diagnosis had been given prior to narcolepsy which is consistent with the prevalence of ADHD in the Norwegian population (1. $(13,14)$. Information regarding family aggregation of neurodevelopmental disorc available for 40 participants (80 \%). In 6 participants (15.0\%) parents reported a 
those without (ADHD total score 18.2 (10.3) versus 18.0 (8.7), $p=0.808$; ADHD ina score 11.3 (7.4) versus 11.1 (5.4), $p=0.582$; ADHD hyperactive/impulsive score 6. versus $6.9(4.8), p=0.996)$.

35Associations between ADHD symptoms and CSF hypocretin-1 level Comparisons between youths with a level of CSF Hcrt-1 below $40 \mathrm{pg} / \mathrm{ml}$ and yout Hcrt-1 level in the range of $40-150 \mathrm{pg} / \mathrm{ml}$ revealed no significant differences in th total score (18.5 (8.4) vs. $16.4(10.6) ; p=0.410)$, inattentive score $(11.8(5.7)$ vs. $\subseteq$ 0.120), or hyperactive/impulsivity score (6.7 (4.7) vs. 7.05.0); $p=0.786$ ).

Within the group of 21 youths with CSF Hcrt-1 level of $40-150 \mathrm{pg} / \mathrm{ml}$ there were r significant correlations between CSF Hcrt-1 level and ADHD total score ( $r h o=-0 . \approx$ 0.169 ), inattentive score ( $r h o=-0.23 ; p=0.312)$, or hyperactive/impulsivity score $0.28 ; p=0.229)$.

Please insert table 2 about here

\subsection{Associations between ADHD symptoms and sleepiness}

The ADHD total and inattentive subscores (but not the hyperactivity/impulsivity : were significantly correlated with parent-reported ESS scores (Table 2). Neither $f$ inattentive, nor hyperactivity/impulsivity subscores were correlated with self-ref scores (Table 2). The association between parent-reported ESS and ADHD total si not significant in univariate or bivariate linear regression analyses (Table 3). 
There were no significant associations between ADHD scores and objective dayti sleepiness (mean MSLT SL) with regard to Spearman correlation (Table 2), univar regression, or multiple linear regression adjusted for age and gender, $\mathrm{AHI}$ and $\mathrm{PL}$

(Table 3).3.7 Associations between ADHD symptoms and sleep fragmentation

There were no significant associations between ADHD total, inattentive or hyperactive/impulsivity scores on the one hand, and sleep stage shift index or an index on the other, either in terms of correlations (Table 2), or in multiple linear I analyses adjusted for age and gender (step 2, Table 3), or for age, gender, AHI, ar index (step 3, Table 3).

Please insert table 3 about here

\section{DISCUSSION}

We report for the first time that a sample of unmedicated youths with post-H1N: of them vaccinated with Pandemrix ${ }^{\otimes}$ ) exhibit a high level of ADHD symptoms, esץ inattention, but also of hyperactivity/impulsivity. Compared with previous report scores in our sample were significantly and considerably higher than in mainly $\mathrm{m}$ narcolepsy youths (9) and in healthy controls (40), but lower than in youths with 40) Moreover, we found that half of the unmedicated NT1 youths had clinically s levels of ADHD symptoms, twice as many as previously reported in the predomin 
from well characterized European mainly NT1 populations defined by internation and using the same ADHD RS. Many drugs that are used to treat narcolepsy are a reduce ADHD symptoms (i.e. methylphenidate or dexamphetamine). Based on tr reasonable to postulate that our prevalence estimate of ADHD symptoms in unm NT1 youths is a more unbiased estimator of the true prevalence than those previ reported from mainly medicated pediatric narcolepsy populations. On the other (18.0\%) youths had no ADHD symptoms, implying that ADHD symptoms are not $\mathrm{i}$ comorbid or co-existing in NT1. Neither age at inclusion, age of disease onset, ag vaccination, gender nor disease duration were associated with the presence of $A$ symptoms, The prevalence of ADHD diagnoses before narcolepsy onset was com the Norwegian population prevalence estimates $(13,14)$, and the ADHD scores $d$ between youths with a family history of neurodevelopmental disorder compared without. Hence, the high occurrence of ADHD symptoms in our sample was not $e$ a high prevalence of pre-existing ADHD diagnose, or a family history of neurodev disorder. ,

The lack of any age or gender influence on ADHD RS scores in our NT1 sample su| the ADHD symptoms in our youths do not represent a neurodevelopmental disor these disorders are characterized by a higher prevalence in males, and with a der symptom load with age (47).

We did not find an association between CSF Hcrt-1 level and ADHD symptoms, in with previous studies $(9,26,27)$. However, others have found associations betw€ 
we used to analyze CSF Hcrt-1 in our study has a lower LOQ of $40 \mathrm{pg} / \mathrm{ml}$, and a va CSF Hcrt-1 level below this limit could be of significance in relation to ADHD symp Secondly, the CSF Hcrt-1 levels and the ADHD assessments were not analyzed simultaneously, since the majority of participants ( $88 \%$ of cases) had a lumbar pı before inclusion in our study. This implies that some participants may have had $\mathrm{f}_{\mathrm{i}}$ decline in CSF Hcrt-1 level between the time of measurement to the time of the / assessment, as is known to occur in some narcolepsy patients $(50,51)$. In one stı adult narcolepsy patients with cataplexy experienced a decline with disease dura However, these narcolepsy patients were atypical in having a normal or intermec Hcrt-1 level when first measured, in contrast to our typical NT1 sample, in which CSF Hcrt-1 levels were low. Furthermore, no correlation between CSF Hcrt-1 leve disease duration was reported in a sample of pediatric and adult NT 1 patients ( 3 as hypocretin neurons project to, and exert their attention-promoting activity via that are directly involved in attention and activity regulation, the ADHD symptor the result of an interaction between low hypocretin output and a relative subclin dysfunction that existed in these other neurons before the debut of the narcolep

We found a weak but significant correlation between parental reports of sleepin ADHD symptoms, but no significant association between self-reported sleepiness symptoms. Youths with no ADHD symptoms had significantly lower self-reportec parent-reported sleepiness compared with those with ADHD symptoms. Divergin 
implying that subjective sleepiness and ADHD symptoms were reported indepen our findings do not support a theory that the overlap of ADHD symptoms and sul sleepiness in narcolepsy is caused by a difficulty in teasing out sleepiness from AI symptoms $(52,55)$. Clinically this implies that a narrow focus on ameliorating day sleepiness when treating narcolepsy carries the risk of overlooking clinically signi impairments in inattention, impulsivity, and hyperactivity.

To our knowledge, our study is also the first to systematically compare simultane assessed ADHD symptoms and subjectively and objectively measured daytime sle unmedicated youths with narcolepsy. In contrast to the subjective measures of $d$ sleepiness, we found no association between ADHD symptoms and the objective sleepiness (MSLT SL). This is in contrast to previous reports of associations betwe ADHD diagnose and shorter MSLT SL $(18,55)$, and also a report of poorer perforn tests of attentional functioning and shorter MSLT SL in unmedicated adult narcol patients (56). These discrepancies towards our findings may be explained by diffe the studied populations (18), inclusion of children with other sleep disorders that (55), and different measures of inattention used (attention test vs. subjective rat since these measures have been reported to have different associations with obj parameters (57). Nevertheless, our results do support previous findings in pediat adult narcolepsy studies (27), and are corroborated by the study of Ramm et al, v no association between performance on the attention test and MSLT SL in a mixe unmedicated adult with central disorders of hypersomnolence (58). 
reporters of both ADHD symptoms and sleepiness, and in addition were not blinc narcolepsy status. Another explanation to the subjective-objective discrepancy $\mathrm{r}$ the two measures in question, the ESS and the MSLT, assess different aspects of regulation (53) that may be differently associated with ADHD symptoms. While t| measures a tendency to fall asleep in various situations, including circumstances person would naturally try to stay awake, the MSLT measures a propensity to fall given situation (in a dark quiet room). This interpretation is supported by the finc our sample, correlations between ESS scores and the MSLT SL were weak and no (data not shown), as has been reported in other studies (53). Given that the ESS includes items measuring the ability to stay awake, one could speculate whether maintenance of wakefulness test (MWT) and the ESS would correlate better thar and the MSLT. Correlations between MWT and ESS have, however, been reporte weak to moderate indicating that they measure different aspects of sleepiness $\mathrm{t}$ way as MSLT and ESS $(60,61)$. Future studies on the association between ADHD s and daytime sleepiness should preferably include the MWT in addition to the ESs MSLT in order to explore this association offering a more complete description ot sleepiness.

Turning to the role of nocturnal sleep fragmentation, ADHD symptoms were not with PSG sleep stage shifts, the number of awakenings, and adjusting for AHI anc did not alter the results. To the best of our knowledge, the association between / symptoms and objective measures of sleep fragmentation has not previously be€ 
different measures of sleep fragmentation from those we used here (sleep efficif arousals, wake after sleep onset).

We report a high occurrence of PLM in our population, in accordance with other: narcolepsy populations, including children (64). Findings of different periodicity a PLMs in narcolepsy compared to PLMs in restless legs syndrome (RLS) (64) and a: between CSF Hcrt-1 level and PLM index in narcolepsy (65) suggest a different pathophysiology of PLMs in narcolepsy, considered to be more related to a gene। dysfunctional tonus regulation in NT1 than related to iron deficiency as in PLMs i 65). All the while, as serum or CSF ferritin has not been studied in relation to PLN narcolepsy $(9,64)$, and ADHD symptoms are associated with iron deficiency (66), iron deficiency for ADHD symptoms in narcolepsy, especially related to PLM, is ir interest and should be investigated in future studies.

Rebound effect with increase of especially hyperactive/impulsive symptoms has described in children with ADHD, (67)_as well as increased amount of REM sleep : discontinuation of anticataplectic medication (68). However, the effect of stimul off quickly (69), and rebound effect are typically reported the same day as medic discontinuation (67), hence in our opinion, it is unlikely that withdrawal of stimul lead to inflated ratings of ADHD symptoms after 14 days off stimulant medicatior extent as to significantly influence our findings.

In our cohort, anticataplectic drugs were additionally tapered off 1-2 weeks prior unmedicated neriod. hence verv low levels even prior to the combletelv unmedic 
The relatively small size of our sample means that our findings must be interpret caution. However, our results do not suggest that ADHD symptoms in narcolepsy explained by the core elements of the narcoleptic phenotype, either low CSF Hcr objectively measured daytime sleepiness, or nocturnal sleep fragmentation. In oI detect significant, but smaller effects of these core elements on ADHD symptoms narcolepsy, larger or pooled samples would be needed. The lack of association bi ADHD symptoms and objective sleep parameters found in our study may also be standard PSG and MSLT measures fail to capture aspects of sleep that could be a with both narcolepsy and ADHD symptomatology, for instance sleep microstruct। deviations such as alterations in cyclically alternating patterns $(70,71)$ or spindle $(72,73)$. Several cognitive dysfunctions underpin the ADHD behavioral symptoms may differ in their relation to hypocretin deficiency or objectively measured slee। characteristics. Various studies, including this one, have featured relatively small and may be heterogeneous with respect to underlying cognitive dysfunctions. St। larger samples including objective measures of cognitive functioning are needed these matters.

In conclusion, we found that symptoms of inattention, hyperactivity, and impulsi prevalent in unmedicated youths with post-H1N1 NT1, and more so than in previ from mainly medicated pediatric narcolepsy patients. Further, the symptoms we। associated with subjective ratings of sleepiness, but not with an ADHD diagnosis 
regarded merely as secondary to sleepiness or sleep fragmentation. Our findings the importance of assessing and treating ADHD symptoms in NT1 separately.

\section{LIMITATIONS}

The results of our study must be interpreted in light of its limitations, the most in which is the lack of a control group. In an attempt to ameliorate some of this dra have we have used norms for the ADHD RS from a culturally, linguistically and socioeconomically similar population (39), and compared our findings with those large Spanish population study of ADHD symptom distribution (40). However, sel significant variables may vary between these population based groups and our gI participants. Participants were not treatment naïve, and measurements of serun ferritin were not available. Also, caution should be used in interpreting our findin symptoms were measured by a questionnaire, and not a clinical diagnosis of ADr addition, ADHD diagnose prior to narcolepsy debut was assessed retrospectively, by combining information from both parental interview and careful review of the patient charts of all participants, and family aggregation of any neurodevelopmel disorder was assessed by parent report and interview by questionnaires and not interview of the individual proband. Further, our study is cross-sectional, so caus be inferred. Finally, we used non-parametric tests to measure correlations, whick undue influence of outliers, but may reduce the ability to detect genuine associat However, parametric correlation estimates did not produce results that altered $\mathrm{t}$ 


\section{ACKNOWLEDGEMENTS}

This study was made possible by the generous help of participating patients and families. We also thank Sleep Technician and Special Nurse Janita Vevelstad for $\mathrm{h}$ work in administering and scoring the sleep recordings, Professor Patricia Howlin critical reading of the manuscript, and Candidatus Scientiarum Jonas Gjesvik for s support.

\section{FUNDING:}

The study has been funded by research support from the Norwegian Competenc Sleep Disorders, the Norwegian Ministry of Health and Care Services, and by a So Norway regional health authority grant (2017070). The funders had no involvem design, collecting, analysis, interpretation of data or writing of report.

Non-financial disclosure $\mathrm{BHH}, \mathrm{HJ}, \mathrm{SEHN}, \mathrm{RV}, \mathrm{PMT}, \mathrm{KBN}, \mathrm{DS}$, and TN have no non-f interests to disclose. SKH has been an expert consultant for the Norwegian state. 


\section{REFERENCES}

1. Kornum BR, Knudsen S, Ollila HM, et al. Narcolepsy. Nat Rev. 2017 Feb 9;3:1610C $10.1038 /$ nrdp. 2016.100 .

2. American Academy of Sleep Medicine (AASM). International Classification of Sle $3^{\text {rd }}$ ed. Darien IL: American Academy of Sleep Medicine; 2014.

3. Pizza F, Magnani M, Indrio C, et al. The hypocretin system and psychiatric disord Psychiatry Rep 2014;16(2):433. doi 10.1007/s11920-013-0433-9.

4. Ohayon MM, Priest RG, Zulley J, et al. Prevalence of narcolepsy symptomatology diagnosis in the European general population. Neurology. 2002;58 (12):1826-33. doi: 10.1212/wnl.58.12.1826

5. Silber MH, Krahn LE, Olson EJ, et al. The epidemiology of narcolepsy in Olmsted ( Minnesota: a population-based study. Sleep. 2002;25(2):197-202. doi: 10.1093/sleep/25 6. Heier MS, Gautvik KM, Wannag E, et al. Incidence of narcolepsy in Norwegian ch adolescents after vaccination against H1N1 influenza A. Sleep Med. 2013;14(9):867-71. c 10.1016/j.sleep.2013.03.020.

7. Partinen M, Saarenpaa-Heikkila O, llveskoski O, et al. Increased incidence and cli of childhood narcolepsy following the $2009 \mathrm{H} 1 \mathrm{~N} 1$ pandemic vaccination campaign in Fin ONE. 2012;7(3). doi: 10.1371/journal.pone.0033723.

8. Miller E, Andrews N, Stellitano L, et al. Risk of narcolepsy in children and young $\mathrm{F}$ receiving AS03 adjuvanted pandemic A/H1N1 2009 influenza vaccine: retrospective anal 2013; Feb 26;346:f794 346 doi: 10.1136/bmj.f794.

9. Lecendreux M, Lavault S, Lopez R, et al. Attention-Deficit/Hyperactivity Disorder symptoms in pediatric narcolepsy: a cross-sectional study. Sleep. 2015;38(8):1285-95. dc 
10. Szakaks A, Hallbook T, Tideman P, et al. Psychiatric comorbidity and cognitive pr children with narcolepsy with or without association to the H1N1 influenza vaccination. 2015;38 (4):615-21. doi: 10.5665/sleep.4582.

11. Kim J, Lee G-H, Sung SM, et al. Prevalence of attention deficit hyperactivity symk narcolepsy: a systematic review. Sleep Med. 2020;65:84-8. doi: 10.1016/j.sleep.2019.07. 12. Rocca FL, Finotti E, Pizza F, et al. Psychosocial profile and quality of life in childre narcolepsy: a case-control study. Sleep. 2016;39(7):1389-98. doi: 10.5665/sleep.5970.

13. Heiervang E, Stormark KM, Lundervold AJ, et al. Psychiatric disorders in Norwegi year-olds: An epidemiological survey of prevalence, risk factors, and service use. J Am Ac Adolesc Psychiatry. 2007;46(4):438-47. doi: 10.1097/chi.0b013e31803062bf.

14. Ullebø AK, Posserud M-J, Heiervang E, et al. Prevalence of the ADHD phenotype year-old children: effects of informant, gender and non-participation. Soc Psychiatry Psy Epidemiol 2012;47(5):763-9. doi: 10.1007/s00127-011-0379-3.

15. Sharma A, Couture, J. A review of the pathophysiology, etiology, and treatment c deficit hyperactivity disorder (ADHD). Ann Pharmacother. 2014;48(2):209-25. doi: $10.1177 / 1060028013510699$.

16. Cortese S, Faraone SV, Konofal E, et al. Sleep in children with attention deficit-hy disorder: meta-analysis of subjective and objective studies. J Am Acad Child Adolesc Psy 2009;48(9):894-908. doi: 10.1097/CHI.0b013e3181ac09c9.

17. Sadeh A, Pergamin L, Bar-Haim, Y. Sleep in children with attention-deficit hypera disorder: A meta-analysis of polysomnographic studies. Sleep Med Rev 2006;10:381-98. 10.1016/j.smrv.2006.03.004.

18. Lecendreux M, Konofal E, Bouvard M, et al. Sleep and alertness in children with ADF 
20. Wajszilber D, Santiseban JA, Gruber, R. Sleep disorders in with ADHD: impact an management challenges. Nat Sci Sleep. 2018;10:453-48. doi: 10.2147/NSS.S163074.

21. Sonuga-Barke EJS, Coghill, D. Editorial Perspective: Laying the foundations for ne generation models of ADHD neuropsychology. J Child Psychol Psychiatry. 2014; 55(11):1: $10.1111 /$ jcpp.12341.

22. American Psychiatric Association (AAP). Diagnostic and Statistical Manual of Mel Disorders, $5^{\text {th }}$ ed. Arlington VA: American Psychiatric Association; 2013.

23. Cortese S, Adamo N, Del Giovane C, et al. Comparative efficacy and tolerability o medications for attention-deficit hyperactivity disorder in children, adolescents, and adı systematic review and network meta-analysis. Lancet Psychiatry. 2018;5(9):727-38. doi: $10.1016 /$ S2215-0366(18)30269-4.

24. Modestino E, Winchester J. A retrospective survey of childhood ADHD symptom among adult narcoleptics. J Att Disord. 2013;17(7):574-82. doi: 10.1177/108705471348C 25. Morse AM, Sanjeev, K. Narcolepsy and psychiatric disorders: Comorbidities or sl pathophysiology? Med Sci. 2018 Feb 15;6(1). pii: E16. doi: 10.3390/medsci6010016.

26. Lopez R, Micoulaud-Franchi J-A, Camodeca L, et al. Association of inattention, hy and hypersomnolence in two clinic-based adult cohorts. J Atten Disord. 2020 Feb;24(4):: doi:10.1177/1087054718775826.

27. Filardi M, Pizza F, Tonetti L, et al. Attention impairments and ADHD symptoms in narcoleptic with and without hypocretin deficiency. PLOS ONE 2017; August 1. doi: 10.1371/journal.pone.0182085.

28. Faraone SV. Advances in the genetics of attention-deficit/hyperactivity disorder. Psychiatry. 2014;76(8):599-600. doi: 10.1016/j.biopsych.2014.07.016. 
10.1093/sleep/28.1.113. 30. Anic-Labat S, Guilleminault C, Kraemer HC, et al. Validati cataplexy questionnaire in 983 sleep-disorders. Sleep. 1999;22(1):77e87.

31. Barkley, R. A., \& Murphy, K. R. (1998). Attention-deficit hyperactivity disorder: A workbook. New York: Guilford Press.

32. Juvodden HT, Alnæs D, Lund MJ, et al. Widespread white matter changes in postnarcolepsy type 1 and first-degree relatives. Sleep. 2018;41(10). doi: 10.1093/sleep/zsy1 33. American Academy of Sleep Medicine. (AASM) Internation Classification of Sleep diagnostic and coding manual. 2 ed: Westchester, IL: American Academy of Sleep Medic 34. Knudsen S, Jennum PJ, Alving J, et al. Validation of the ICSD-2 criteria for CSF hyF measurements in the diagnosis of narcolepsy in the Danish population. Sleep. 2010;33(2 doi: 10.1093/sleep/33.2.169.

35. Gomez, R. (2007). Australian parent and teacher ratings of the DSMIV ADHD syr differential symptom functioning and parent-teacher agreement and differences. J Atter 2007; 11(1), 17-27. doi: 10.1177/1087054706295665.

36. Kornør, H \& Bøe, T. Psychometric properties of the Norwegian version of ADHDRating scale - IV Home version (ADHD-RS-IV Home). PsykTestBarn.2011;1:8.

37. Dopfner M, Steinhausen $\mathrm{H}-\mathrm{C}$, Coghill D, et al. Cross-cultural reliability and validit assessed by the ADHD Rating Scale in a pan-European study. Eur Child Adolesc Psychiatr 15:I/46-I/55 (2006) doi: 10.1007/s00787-006-1007-8.

38. Szomlaiski N, Dyrborg J, Rasmussen $\mathrm{H}$, et al. Validity and clinical feasibility of the scale (ADHD-RS) A Danish Nationwide Multicenter Study. Acta Paed. 2009;98(2):397-402 10.1111/j.1651-2227.2008.01025.x.

39. Poulsen L, Jørgensen S, Dalsgaard S, et al. Danish standardization of the attentio 
40. Vallejo-Valdivielsoa M, Soutulloa CA, de Castro-Manglanoa P, et al. Validation of language version of the ADHD Rating Scale IV in a Spanish sample. Neurología. 2019; No' Dec;34(9):563-72. doi: 10.1016/j.nrl.2017.05.010.

41. Nordstrand SHE, Hansen BH, Rootwelt T, et al. Psychiatric symptoms in with po H1N1 narcolepsy type 1 in Norway.Sleep.2019 Apr 1;42(4) doi: 10.1093/sleep/zsz008.

42. Achenbach TM, Rescorla L. Manual for the ASEBA School- Age Forms \& Profiles: 1 Behavior Checklist for Ages 6-18, Teacher's Report Form, Youth Self-Report: An Integrat Multi-Informant Assessment. Burlington, VT: ASEBA; 2001.

43. Achenback T, Rescorla L. Manual for the ASEBA Adult Forms \& Profiles. Burlingtc University of Vermont, Research Center for Children, Youth, \& Families; 2003.

44. Novik TS. Validity of the child behaviour checklist in a Norwegian sample. Eur Chi Psychiatry. 1999;8 (4):247-54. doi: 10.1007/s007870050098.

45. Johns MW. A new method for measuring daytime sleepiness: the Epworth sleep Sleep. 1991;14(6):540-45. doi: 10.1093/sleep/14.6.540.

46. Field A. Discovering Statistics Using SPSS. London: Sage Publications; 2009.

47. Soendergaard HM, Hove Thomsen P, Pedersen E, et al. Associations of age, gend subtypes with ADHD symptoms and related comorbidity in a Danish sample of clinically I adults. J Atten Disord. 2016;20(11):925-33. doi: 10.1177/1087054713517544.

48. Hansen BH, Skirbekk S, Oerbeck B, et al. Associations between sleep problems aı attentional and behavioral functioning in children with anxiety disorders and ADHD. Beh Med. 2014;12:53-68. doi: 10.1080/15402002.2013.764525.

49. Booth JE, Carlson CL, Tucker DM. Performance on a neurocognitive measure of a 
50. Lopez R, Barateau L, Evangelista E, et al. Temporal changes in the cerebrospinal hypocretin-1 and histamine in narcolepsy. Sleep. 2017;40(1). doi: 10.1093/sleep/zsw010 51. Pizza F, S V, Liguori R, Parchi P, et al. Primary progressive narcolepsy type 1: The the coin. Neurology. 2014 Dec 2;83(23):2189-90. doi: 10.1212/WNL.0000000000001051

52. Oosterloo M, Lammers GJ, Overeem S, et al. Possible confusion between primar hypersomnia and adult attention-deficit/hyperactivity disorder. Psychiatry Res 143 (2001 10.1016/j.psychres.2006.02.009.

53. Sangal RB, Thomas L, Mitler MM. Maintenance of wakefulness test and multiple slee test. Measurement of different abilities in with sleep disorders. Chest. 1992;101(4):898doi:10.1378/chest.101.4.898.

54. Ito W, Honda M, Ueno T, et al. Hypersomnia with ADHD: a possible subtype of narco Sleep Biol Rhythms (2018) 16:205-10. https://doi.org/10.1007/s41105-017-0139-1.

55. Golan N, Shahar E, Ravid S, et al. Sleep Disorders and daytime Sleepiness in children attention-deficit/hyperactivity disorder. Sleep.2004;27(2): 261-6. doi: 10.1093/sleep/27. 56. Bayard S, Langenier M, De Cock V, et al. Executive control of attention in narcole ONE 2012;7(4). doi: 10.1371/journal.pone.0033525.

57. Zamarian L, Högl B, Delazer M, et al. Subjective deficits of attention, cognition ar depression in with narcolepsy. Sleep Med. 2015;16:45-51. doi: 10.1016/j.sleep.2014.07. 58. Ramm M, Rafarpour A, Boentert M, et al. The Perception and Attention Functior battery as a measure of neurocognitive impairment in with suspected central disorders hypersomnolence. J Sleep Res. 2018;27:275-82. doi: 10.1111/jsr.12587.

59. Sangal RB, Mitler MM, Sangal JM., Subjective sleepiness ratings (Epworth sleepin 
60. Erman M, Emsellem H, Black J et al. Correlation between the Epworth Sleepiness the Maintenance of Wakefulness Test in with narcolepsy participating in two clinical tric oxybate. Sleep Med 2017; : 92-5.

61. Walters A, Silvestri R, Zucconi M, et al. Review of the possible relationship and $\mathrm{h}$ links between attention deficit hyperactivity disorder (ADHD) and the simple sleep relat disorders, parasomnias, hypersomnias, and circadian rhythm disorders. J Clin Sleep Med $2008 ; 4(6): 591-600$.

62. Sadeh A, Gruber R, Raviv A. Sleep, neurobehavioral functioning, and behavior pr school-age children. Child Dev. 2002;73(2):405-17. doi: 10.1111/1467-8624.00414.

63. Blunden S, Beebe D. The contribution of intermittent hypoxia, sleep debt and sle disruption to daytime performance deficits in children: Consideration of respiratory and repsiratory sleep disorders. Sleep Med Rev. 2006;10:109-18. doi: 10.1016/j.smrv.2005.1

64. Ferri R, DelRosso LM, Aricò D, et al. Leg movement activity during sleep in scho children and adolescents: a detailed study in normal controls and participants with restl syndrome and narcolepsy type 1 . Sleep. 2018;41(4). doi: 10.1093/sleep/zsy010.

65. Knudsen S, Gammeltoft S, Jennum PJ. Rapid eye movement sleep behavior disor narcolepsy is associated with hypocretin deficiency. Brain 2010: 133; 568-79. doi: 10.1093/brain/awp320.

66. Tseng PT, Cheng YS, Yen CF, et al. Peripheral iron levels in children with attentic hyperactivity disorder: a systematic review and meta-analysis. Sci Rep. 2018;8(1):788. dc 10.1038/s41598-017-19096-x.

67. López FA, Childress A, Adeyi B, et al. ADHD Symptom Rebound and Emotional La Lisdexamfetamine Dimesylate in Children Aged 6 to 12 Years. J Atten Disord. 2017;21(1) 
68. Feige B, Voderholzer U, Riemann D, et al. Fluoxetine and sleep EEG: effects of a : subchronic treatment, and discontinuation in healthy subjects. Neuropsychopharmacol. 2002;26(2):246-58. doi: 10.1016/S0893-133X(01)00314-1.

69. Zeiner P. Do the beneficial effects of extended methylphenidate treatment in bc attention-deficit hyperactivity disorder dissipate rapidly during placebo treatment? Norc 1999;53(1):55-60. doi.org/10.1080/080394899426738.

70. Ferri R, Franceschini C, Zucconi M, et al. Sleep polygraphic study of children and with narcolepsy/cataplexy. Dev Neuropsychol 2009;34(5):523-38. doi: 10.1080/8756564 71. Akinc G, Oztura I, Hiz S, et al. Sleep structure in children with attention-deficit/h disorder. J Child Neurol 2015;30(11):1520-5. doi: 10.1177/0883073815573318.

72. Christensen JAE, NikolicM, Hvidtfelt M, et al. Sleep spindle density in narcolepsy. 2017;34:40-9. doi: 10.1016/j.sleep.2017.02.022.

73. Merikanto I, Kuulaa L, Makkonena T, et al. ADHD symptoms are associated with d activity of fast sleep spindles and poorer procedural overnight learning during adolescen Neurobiol Learn Mem 2019;57:106-13. doi: 10.1016/j.nlm.2018.12.004. 